PREPARED FOR SUBMission to JHEP

\title{
Effect of $r$ averaging on Chiral Anomaly in Lattice QCD with Wilson Fermion: Finite volume and cutoff effects
}

\author{
Asit K. De, A. Harindranath and Santanu Mondal \\ Theory Division, Saha Institute of Nuclear Physics \\ 1/AF Bidhan Nagar, Kolkata 700064, India \\ E-mail: asitk.de@saha.ac.in, a.harindranath@saha.ac.in, \\ santanu.mondal@saha.ac.in
}

\begin{abstract}
We demonstrate the effectiveness of averaging over the Wilson parameter $r$ (which has been proposed earlier) in removing the cutoff effects of naive Wilson fermions in both the anomaly term and the pseudoscalar density term in the flavor singlet axial Ward Takahashi identity at $\mathcal{O}\left(g^{2}\right)$ involving slowly varying background gauge fields. We show that it is the physical fermion contribution which is largely influenced by the $r$ averaging. We have studied the possible interplay between finite size and cutoff effects by investigating in detail naive, $\mathcal{O}(a)$ improved and OStm Wilson fermion cases for a range of volumes and lattice fermion mass $(\mathrm{am})$. For naive Wilson fermions $r$ averaging is shown to remove the effects of the interplay. We have shown that for the pseudoscalar density term to $\mathcal{O}\left(g^{2}\right)$ the lattice result differs from the continuum result by exhibiting considerable am dependence which appears to be a manifestation of cutoff effects with naive Wilson fermion. The pseudoscalar density term to $\mathcal{O}\left(g^{2}\right)$ is shown to be almost independent of am when $r$-averaging is performed.
\end{abstract}




\section{Contents}

1 Introduction $\quad 1$

2 Naive, $\mathcal{O}(a)$ improved and OStm Wilson Fermions 3

3 Wilson parameter $r$ Averaging $\quad 5$

4 Anomaly: contribution from different regions of the Brillouin zone 5

5 Volume Dependence $\quad 6$

$\begin{array}{lll}6 & \text { Pseudoscalar density term versus the anomaly term } & 8\end{array}$

$\begin{array}{lll}7 & \text { Conclusions } & 11\end{array}$

\section{Introduction}

Recently we have studied [1] the emergence of the chiral anomaly in the continuum chiral limit with Osterwalder-Seiler twisted mass (OStm) Wilson fermions [2] in lattice QCD in comparison with naive [3], $\mathcal{O}(a)$, and $\mathcal{O}\left(a^{2}\right)$ improved [4-6] Wilson fermions. Here $a$ denotes the lattice spacing. Karsten and Smit [7], Kerler [8, 9] and Seiler and Stamatescu [10] have demonstrated some time ago that naive Wilson fermions on the lattice can indeed reproduce the chiral anomaly in the infinite volume chiral limit in the continuum. However for a lattice simulation, understanding the effects of the finite volume, finite cutoff and nonzero fermion mass is very crucial. Possible interplay among various lattice artifacts in the unimproved theory and how various suggested improvements help to ameliorate the situation also needs to be investigated. Lattice calculation of the chiral anomaly to one loop perturbation theory with finite lattice fermion mass $(\mathrm{am})$ and finite box size $(L)$ provides an excellent laboratory to address some of these issues in a simple setting.

Aoki [11] proposed taking the average over the Wilson parameter $r= \pm 1$ for improving the scaling behavior of chiral condensate. David and Hamber [12] also proposed similar ideas. For a detailed discussion of the symmetries associated with $r \rightarrow-r$ and their implications, specifically that the $r$ averaged correlators are affected by only $\mathcal{O}\left(\left(a^{2}\right)^{k}\right), k=$ $1,2, \ldots$, discretization effects, see Frezzotti and Rossi [13]. Twisted mass (tm) fermion [14-16] has an effective $r$ averaging, but at finite lattice spacing there are isospin breaking cut off artifacts. However, in all simulations carried out until now, $\mathcal{O}\left(a^{2}\right)$ effects have been shown to be under control and to disappear in the continuum limit. Large $\mathcal{O}\left(a^{2}\right)$ effects are only visible in the neutral pion mass [17]. This observation has been discussed in detail and explained theoretically in Ref. [18]. 
We note in passing that since positivity property of the quark determinant is not satisfied by the OStm Wilson (W) QCD action, numerical simulation is only possible with a mixed action with OStm WQCD action for the valence quarks and tm WQCD action for the sea quarks.

$r$ averaging of lattice QCD with naive Wilson fermions looks interesting. The flavour singlet axial anomaly in this case can be studied analytically under certain assumptions and the effect of the $r$ averaging investigated quantitatively for each of the terms on the right hand side of the Ward Takahashi identity. In this work there is overwhelming evidence for improvement with respect to cutoff effects, once $r$ averaging is done. In fact the $r$ averaged result is much better than $\mathcal{O}\left(a^{2}\right)$ improved Wilson fermion with tree level coefficients [4]. In addition there are interesting issues of interplay between $\mathcal{O}(a)$ cutoff effects and finite volume effects, and its removal after $r$ averaging. Contributions from the physical fermion and the different doubler fermions are also considered separately. Finally the effect of $r$ averaging on the pseudoscalar density (PSD) term on the right hand side of the Ward Takahashi identity is also studied. In the following paragraphs of the introduction we briefly discuss some of these issues.

Since the emergence of anomaly is intimately tied with the removal of fermion doublers, the behavior of their contributions as one varies the parameters of the theory is of interest. Recall that as the Wilson parameter $r$ is set to zero, the anomaly contributions from the 16 species (the physical fermion and the 15 doublers) all cancel each other. Karsten and Smit showed that for non-vanishing $r$, anomaly is independent of $r$ in the limit $a \rightarrow 0$. However, on a lattice with finite cutoff (practical situation), doubler masses are not exactly infinitely large and they do contribute to the anomaly depending upon the value of $r$. We study the doubler contribution $[4,7]$ as a function of $r$ and $a m$.

We also compare the finite volume dependence of the one loop calculation of the anomaly with naive Wilson action and the improved actions. The effect of small momentum behavior of the integrand as the fermion mass is lowered is investigated with both Periodic (P) and Anti-Periodic (AP) boundary conditions (BC). We find that the naive Wilson fermion has larger finite volume effects than the improved ones possibly because of the interplay between the finite volume and cutoff effects. Even though OStm Wilson fermion has a better chiral behavior as far as approach to the chiral limit is concerned than the $\mathcal{O}(a)$ improved Wilson fermion in the infinite volume limit [1], we find that with PBC, finite volume effects are more pronounced with the former than with the latter. APBC improves the convergence behavior as $L$ becomes large for both naive and OStm Wilson fermions. The most striking result, however, is that finite volume effects of naive Wilson fermions are largely removed with $r$ averaging, indicating that finite volume effects of naive Wilson fermions observed in this calculations are mostly at $\mathcal{O}(a)$.

At the tree level, in the continuum limit, the flavour singlet axial vector current is conserved in the chiral limit because both the PSD term and the contribution from the Wilson term (denoted by $\chi$ later) vanish. To $\mathcal{O}\left(g^{2}\right)$, in the continuum limit, it can be seen that the PSD term is independent of the fermion mass for a slowly varying background gauge field [19] and produces the negative of the anomaly term, leading to a cancellation. However note that this cancellation is due to the assumption of slowly varying background gauge 
field and at this order only. For general gauge fields and to any order of $g^{2}$, cancellation does not occur and the flavour singlet axial vector Ward Takahashi identity is anomalous. For $\mathcal{O}(a)$ improved Wilson fermions, at $\mathcal{O}\left(g^{2}\right)$ the cancellation has been demonstrated in the chiral limit for constant background gauge field by Hamber and Wu [4]. Our starting point is the flavour singlet axial vector Ward Takahashi identity on the lattice with naive and improved Wilson fermions. Our emphasis is to study the effect of $r$ averaging on the anomaly and the PSD terms individually, as discussed above. In the case of naive Wilson fermions, both the PSD and the anomaly terms individually show strong lattice mass dependence. By using the Reisz's power counting theorem [20] for the PSD term to $\mathcal{O}\left(g^{2}\right)$ for a slowly varying background gauge field we show that the PSD term is independent of fermion mass in the continuum limit. This result indicates that the observed mass dependence with naive Wilson fermion is a pure lattice artifact which is largely removed by $r$ averaging leading to remarkable $a m$-independence.

\section{Naive, $\mathcal{O}(a)$ improved and OStm Wilson Fermions}

The flavor singlet axial Ward Takahashi identity for the naive Wilson fermions on the Euclidean lattice reads

$$
\left\langle\Delta_{\mu}^{b} J_{5 \mu}(x)\right\rangle=2 m\left\langle\bar{\psi}_{x} \gamma_{5} \psi_{x}\right\rangle+\left\langle\chi_{x}\right\rangle
$$

where $\langle\mathcal{O}\rangle$ denotes the functional average of $\mathcal{O}$ for a background gauge field. Explanation of other terms are as follows:

$$
\text { The backward derivative, } \begin{aligned}
\Delta_{\mu}^{b} f(x) & =\frac{1}{a}[f(x)-f(x-\mu)] \\
J_{5 \mu}(x) & =\frac{1}{2}\left[\bar{\psi}_{x} \gamma_{\mu} \gamma_{5} U_{x, \mu} \psi_{x+\mu}+\bar{\psi}_{x+\mu} \gamma_{\mu} \gamma_{5} U_{x \mu}^{\dagger} \psi_{x}\right] \\
\text { and }\left\langle\chi_{x}\right\rangle & =-\operatorname{Trace}\left[\gamma_{5}(G W+W G)\right] .
\end{aligned}
$$

The Green function

$$
G(x, y)=\left\langle x\left|\frac{1}{\left[\gamma_{\mu} D_{\mu}+W+m\right]}\right| y\right\rangle,
$$

where

$$
\begin{aligned}
{\left[D_{\mu}\right]_{x y} } & =\frac{1}{2 a}\left[U_{x, \mu} \delta_{x+\mu, y}-U_{x-\mu, \mu}^{\dagger} \delta_{x-\mu, y}\right] \\
W_{x y} & =\frac{r}{2 a} \sum_{\mu}\left[2 \delta_{x, y}-U_{x, \mu} \delta_{x+\mu, y}-U_{x-\mu, \mu}^{\dagger} \delta_{x-\mu, y}\right] .
\end{aligned}
$$

For ease of comparisons and to facilitate discussions, here we collect the expressions for chiral anomaly in the naive, $\mathcal{O}(a)$ improved and OStm Wilson fermions [1]. 
The contribution to axial vector Ward Takahashi identity from the Wilson term in the unimproved theory

$$
\begin{aligned}
\left\langle\chi_{x}\right\rangle= & 2 g^{2} \epsilon_{\mu \nu \rho \lambda} \operatorname{trace} F_{\mu \nu}(x) F_{\rho \lambda}(x) \frac{1}{(2 \pi)^{4}} \sum_{p} \cos \left(p_{\mu} a\right) \cos \left(p_{\nu} a\right) \cos \left(p_{\rho} a\right) \\
& \times W_{0}(p)\left[\cos \left(p_{\lambda} a\right)\left[m+W_{0}(p)\right]-4 \frac{r}{a} \sin ^{2}\left(p_{\lambda} a\right)\right]\left(\mathcal{G}_{0}(p)\right)^{3} \\
= & \frac{g^{2}}{8(\pi)^{4}} \epsilon_{\mu \nu \rho \lambda} \operatorname{trace} F_{\mu \nu}(x) F_{\rho \lambda}(x) \tilde{I}(a m, r, L)
\end{aligned}
$$

where

$$
\begin{aligned}
W_{0}(p) & =\frac{r}{a} \sum_{\mu}\left[\left(1-\cos \left(a p_{\mu}\right)\right)\right] \\
\mathcal{G}_{0}(p) & =\left(\frac{1}{a^{2}} \sum_{\mu} \sin ^{2}\left(a p_{\mu}\right)+\left(m+\frac{r}{a} \sum_{\mu}\left[1-\cos \left(a p_{\mu}\right)\right]\right)^{2}\right)^{-1}
\end{aligned}
$$

and trace is in color space.

Explicitly, $\sum_{p}=\left(\frac{1}{L}\right)^{4} \sum_{n_{1}, n_{2}, n_{3}, n_{4}}$ where $n_{1}, n_{2}, n_{3}, n_{4}=0,1,2,3, \cdots$. In the infinite volume, chiral limit, $\tilde{I} \rightarrow-\frac{\pi^{2}}{2}$ so that $-\frac{2}{\pi^{2}} \tilde{I}=I \rightarrow 1$. In all our plots it is the function $I(a m, r, L)$ called anomaly integral which we have plotted.

For $\mathcal{O}(a)$ improved Wilson fermion

$$
\begin{aligned}
\left\langle\chi_{x}^{I}\right\rangle=\quad & 2 g^{2} \epsilon_{\mu \nu \rho \lambda} \operatorname{trace} F_{\mu \nu}(x) F_{\rho \lambda}(x) \frac{1}{(2 \pi)^{4}} \sum_{p} \cos \left(a p_{\mu}\right) \cos \left(a p_{\rho}\right) \cos \left(a p_{\lambda}\right)\left[\mathcal{G}_{0}^{I}(p)\right]^{3} \\
& {\left[\cos \left(a p_{\nu}\right)\left[m+W_{0}(p)+W_{0}^{I}(p)\right]\right.} \\
& \left.-4 \frac{r}{a} \sin \left(a p_{\nu}\right)\left(\sin \left(a p_{\nu}\right)-\frac{1}{2} \sin \left(2 a p_{\nu}\right)\right)\right]\left[W_{0}(p)+W_{0}^{I}(p)\right]
\end{aligned}
$$

Here

$$
\begin{array}{r}
W_{0}(p)+W_{0}^{I}(p)=\left[\sum_{\mu}\left[\frac{r}{a}\left(1-\cos \left(a p_{\mu}\right)\right)+\frac{r}{4 a}\left(-1+\cos \left(2 a p_{\mu}\right)\right)\right]\right], \\
\mathcal{G}_{0}^{I}(p)=\left(\frac{1}{a^{2}} \sum_{\mu} \sin ^{2}\left(a p_{\mu}\right)+\left(m+\sum_{\mu}\left[\frac{r}{a}\left[1-\cos \left(a p_{\mu}\right)\right]+\frac{r}{4 a}\left[-1+\cos \left(2 a p_{\mu}\right)\right]\right]\right)^{2}\right)^{-1}
\end{array}
$$

In the case of the OStm Wilson action

$$
\begin{aligned}
\left\langle\chi_{x}^{o s}\right\rangle & =2 g^{2} \epsilon_{\mu \nu \rho \lambda} \operatorname{trace} F_{\mu \nu}(x) F_{\rho \lambda}(x) \frac{1}{(2 \pi)^{4}} \sum_{p} \cos \left(p_{\mu} a\right) \cos \left(p_{\nu} a\right) \cos \left(p_{\rho} a\right) \\
& \times W_{0}(p)\left[\cos \left(p_{\lambda} a\right) W_{0}(p)-4 \frac{r}{a} \sin ^{2}\left(p_{\lambda} a\right)\right]\left(\mathcal{G}_{0}^{o s}(p)\right)^{3}
\end{aligned}
$$

where

$$
\left(\mathcal{G}^{o s}\right)^{-1}=D^{2}-m^{2}-W^{2}
$$




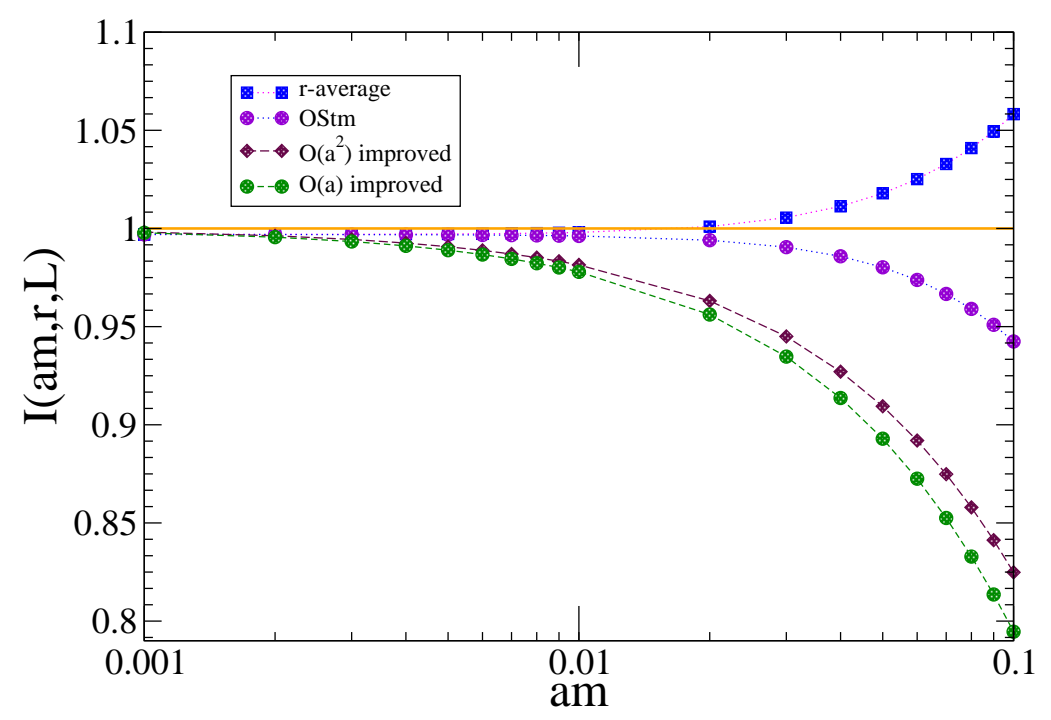

Figure 1. Anomaly integral for $0.001<a m<0.1$ for $r$ averaged naive Wilson fermions at $L=60$ for $r= \pm 1$ compared with OStm, $\mathcal{O}(a)$ and $\mathcal{O}\left(a^{2}\right)$ improved Wilson fermions with $r=1$.

\section{$3 \quad$ Wilson parameter $r$ Averaging}

In Fig. 1 we compare the anomaly integral for $r$ averaged naive Wilson fermion with the OStm, $\mathcal{O}(a)$ and $\mathcal{O}\left(a^{2}\right)$ improved Wilson fermions. The $r$ averaged anomaly integral for naive Wilson fermion shows better chiral behaviour compared with OStm Wilson fermion for $a m \geq 0.01$. The cutoff effects are known to be proportional to even powers of $a$ for both $r$-averaged and OStm Wilson fermions but they are not the same as illustrated in Fig. 1. Although the leading cutoff effect in $r$ averaged naive Wilson fermion and OStm Wilson fermion is of $\mathcal{O}\left(a^{2}\right)$, they have much better chiral behaviour than the $\mathcal{O}\left(a^{2}\right)$ improved [4] Wilson fermion which employs tree level coefficients. For further improvement in the latter case one needs to tune the coefficients while OStm and $r$-averaged Wilson fermion do not need any tuning.

In the following sections we present results of $r$ averaging for the anomaly integral for different lattice volumes and $a m$ and contributions to the integral from different doubler sectors.

\section{Anomaly: contribution from different regions of the Brillouin zone}

It is instructive to separately consider the contribution to the anomaly integral from different regions of the Brillouin zone in order to compare and contrast the contribution of the physical fermion and the doublers as a function of the different parameters. This has been studied in the chiral limit by Hamber and Wu [4]. However, for practical simulations the behavior at non-zero quark masses and nonzero lattice spacing is more relevant and we 
study this issue in this section. Following Karsten and Smit, the limits on the momentum sum are changed from $(-\pi / a,+\pi / a)$ to $(-\pi /(2 a), 3 \pi /(2 a))$ and further the momentum sum hypercube is divided into 16 smaller hypercubes corresponding to $(-\pi /(2 a),+\pi /(2 a))$ and $(+\pi /(2 a),+3 \pi /(2 a))$ for each $p_{\mu}, \mu=1,2,3,4$. Thus the total anomaly contribution is decomposed into the contributions from the five species-types and the Anomaly Integral $I=I_{0}-4 I_{1}+6 I_{2}-4 I_{3}+I_{4}$. In Figs. 2(a) and 2(b) we compare the contributions in the region $0.01<a m<1.0$ for $r=1$ for naive Wilson fermions and OStm Wilson fermions respectively. As expected, as the physical fermion gets lighter, its contribution to the anomaly is dominant compared to the doublers. Nevertheless, doublers' contribution is not insignificant. The first doubler contributes the most since it has the lowest mass among the doublers. Also note that the lowest mass doubler has more am dependence in the case of naive Wilson fermion compared to OStm fermion.

To verify that the doublers do decouple when their masses become very heavy so that the sole contribution to the anomaly comes from the physical fermion, we study the behavior of $I_{i}, i=1,2,3,4$ as the Wilson parameter $r$ is increased. We realize that the reflection positivity is not guaranteed when $|r|>1$, but in the present context we adjust $\mathrm{r}$ only to make the doubler masses heavy.

In Fig. 2(c) we present the total contribution of the doublers to the anomaly integral for $0.01<a m<0.1$ for naive Wilson fermions at $L=40$ for $1 \leq r \leq 2$. As expected, the total doubler contribution diminishes as $r$ increases and it is not very sensitive to $a m$ in the range $0.01 \leq a m \leq 0.1$. Fig. $2(\mathrm{~d})$ shows, for $r= \pm 1$, total doubler contribution and physical fermion contribution separately. For each $r$, total contributions from the doublers have slight am dependence. Although for each $r$, the contribution from the physical fermion depends significantly on am, the figure clearly indicates that, on $r$ averaging, the amdependence will cancel out.

\section{Volume Dependence}

Since numerical simulations are performed at finite volume and finite lattice spacing, it is of interest to study the finite volume dependence of the anomaly integral as a function of the quark mass. Since finite size artifacts are different for the three fermion actions considered in this work, and finite volume and finite cutoff $(1 / a)$ effects may interfere with each other, the anomaly integral provides us an opportunity to explore this issue in this simple setting.

First we discuss the case of PBC in all four directions. In Fig. 3(a) volume dependence of the anomaly integral for $0.01 \leq a m \leq 0.1$ for naive Wilson fermion for $r=1$ and PBC is presented. We note that the behavior qualitatively changes as am changes from large to small. Moreover, irrespective of the quark mass, approximate $L$ independence is achieved only for $L \gtrsim 40$. In Fig. 3(b) the corresponding behavior for $O(a)$ improved Wilson fermion is shown. Here the $L$ dependence is qualitatively the same across the range of $a m$, and furthermore, $L$ independence is achieved for $L>20$. Volume dependence of the anomaly integral for OStm Wilson fermion is presented in Fig. 3(c). Here again the $L$ dependence is qualitatively the same across the mass range but $L$ independence is achieved only for 
(a)

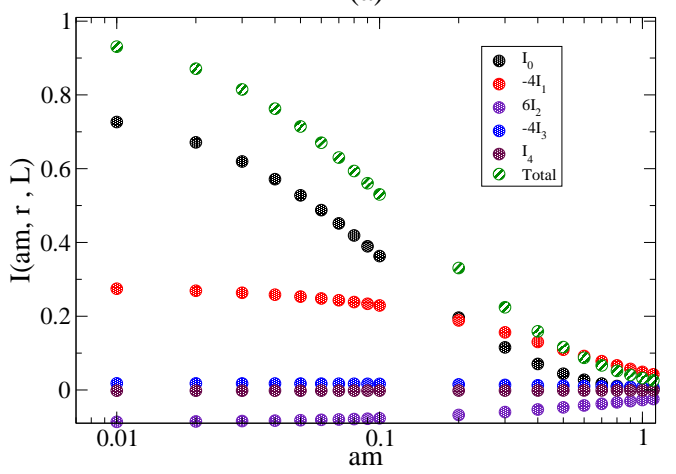

(c)

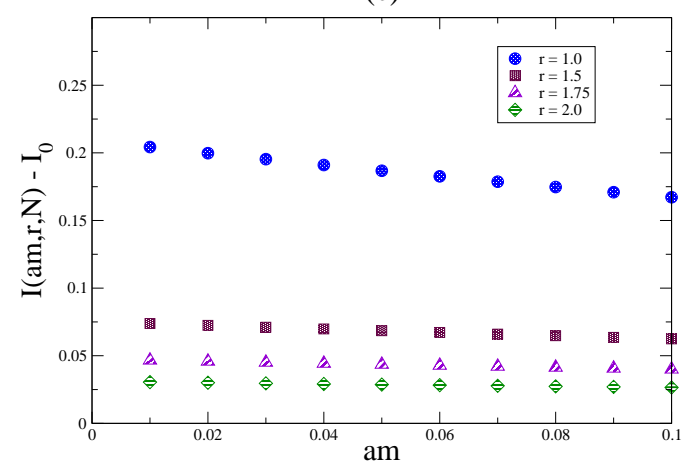

(b)

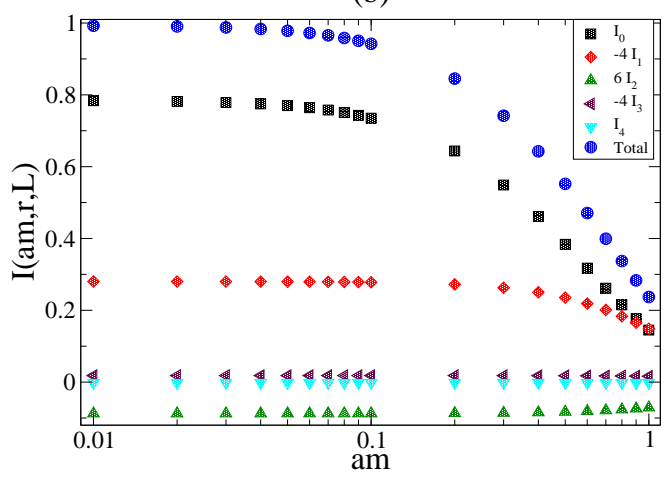

(d)

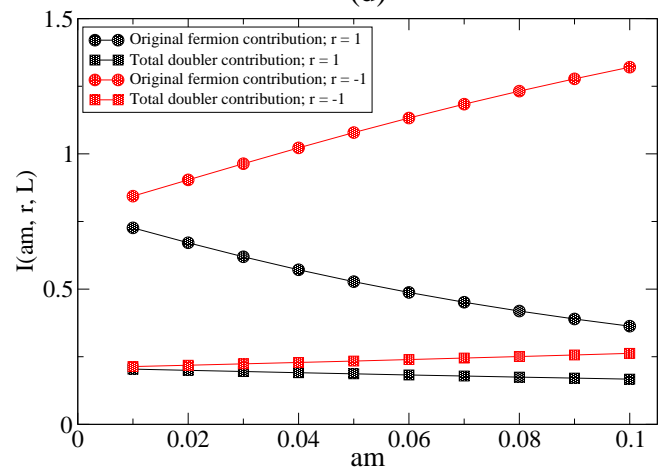

Figure 2. (a) Contributions from different regions of the Brillouin zone to the anomaly integral for $r=1$ and $0.01<a m<1.0$ for naive Wilson fermions at $L=40$ compared with the total contribution. (b) Contributions from different regions of the Brillouin zone to the anomaly integral for $r=1$ and $0.01<a m<1.0$ for OStm Wilson fermions at $L=40$ compared with total contribution. (c) Total contribution of the doublers to the anomaly integral for $0.01<a m<0.1$ for naive Wilson fermions at $L=40$ for $1 \leq r \leq 2$. (d) Comparison of total doubler contribution and the physical fermion contribution for $0.01<a m<0.1$ with naive Wilson fermions at $L=40$ and $r= \pm 1$.

$L>50$. Nevertheless the infinte volume result for OStm fermion is closer to the continuum compared to $\mathcal{O}(a)$ improved result. The small $L$ behavior is different for the three types of fermions considered here.

We have checked the sensitivity with respect to boundary condition of the volume dependence of the anomaly integral for the three actions in Fig. 3(d). For the $O(a)$ improved Wilson fermion, there is sensitivity to boundary condition only for very small $L$. On the other hand both for the naive and OStm Wilson fermion, the APBC improves the convergence of the anomaly integral with respect to $L$.

In Figs. 3(a), 3(b), 3(c) and 3(d) we have seen some interplay between the finite volume 
(a)

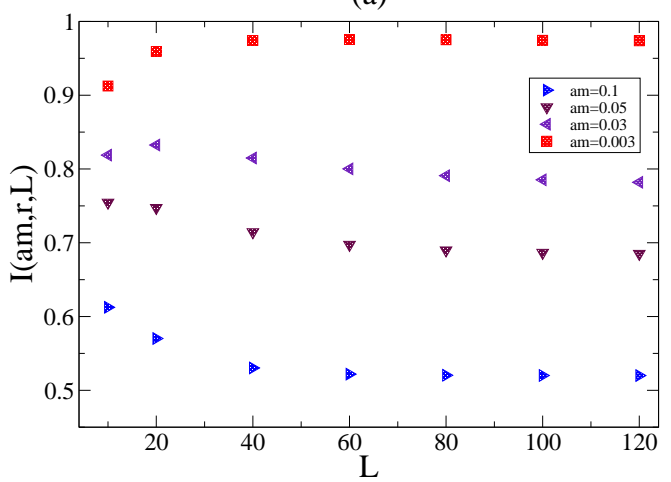

(c)

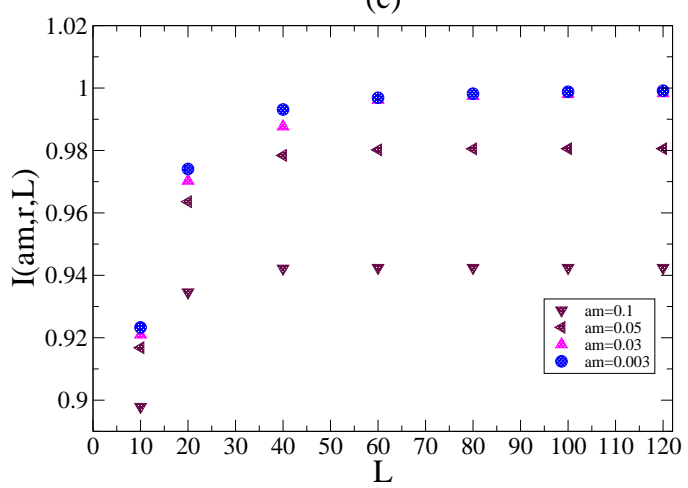

(b)

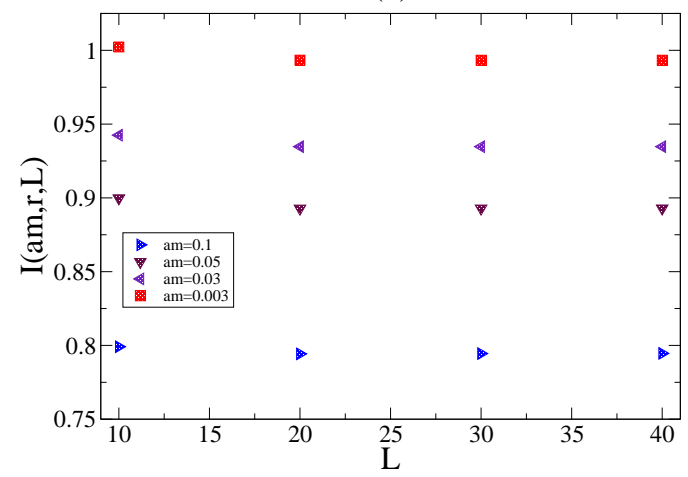

(d)

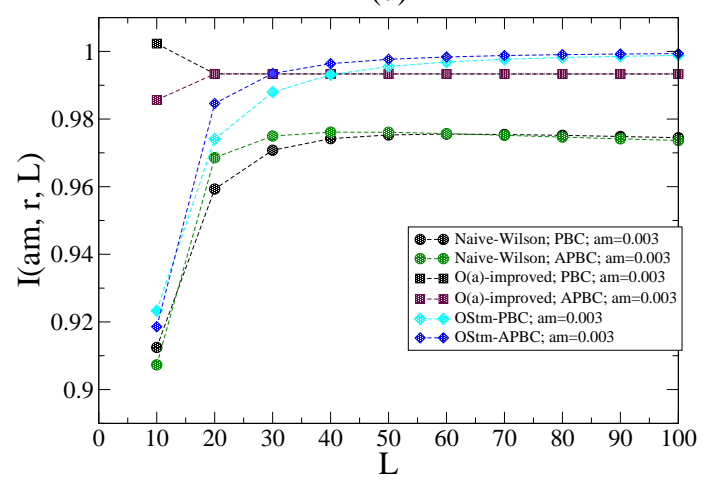

Figure 3. (a) Volume dependence of the anomaly integral for $r=1$ and $0.01 \leq a m \leq 0.1$ for naive Wilson fermions with PBC. (b) Volume dependence of the anomaly integral for $r=1$ and $0.003 \leq a m \leq 0.1$ for $\mathcal{O}(a)$ improved Wilson fermions with PBC. (c) Volume dependence of the anomaly integral for $r=1$ and $0.003 \leq a m \leq 0.1$ for OStm Wilson fermions with PBC. (d) Volume dependence of the anomaly integral for $a m=0.003$ and $10 \leq L \leq 120$ for naive Wilson, $\mathcal{O}(a)$ improved Wilson and OStm Wilson fermions with PBC and APBC.

and the cutoff effects for naive Wilson fermion. The fact that this interplay is absent for $\mathcal{O}(a)$ improved Wilson fermion indicates that this is largely an $\mathcal{O}(a)$ artifact. Finally Fig. 4 shows that the convergence of the anomaly integral with respect to $L$ is vastly improved by $r$ averaging, since it removes the cutoff effects to a great extent.

\section{Pseudoscalar density term versus the anomaly term}

After the detailed analysis of the anomaly integral with respect to the $a m, r$ and $L$ dependence, we revisit the flavor singlet axial Ward Takahashi identity, Eq. (2.1). To $\mathcal{O}\left(g^{2}\right)$, for slowly varying background gauge fields the contribution from the PSD term is 


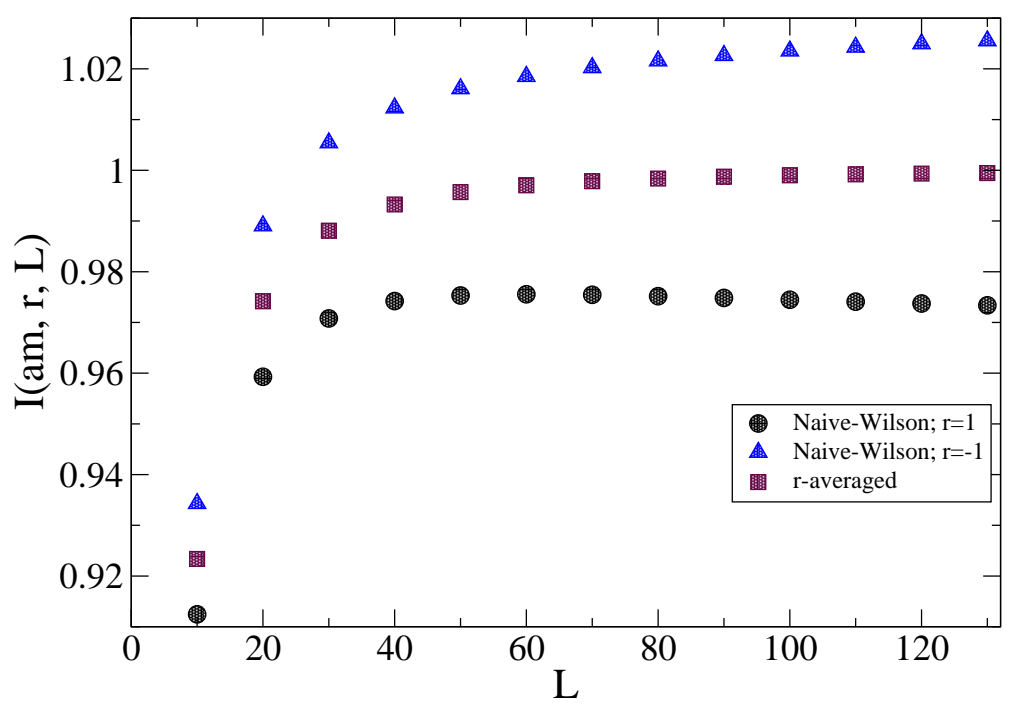

Figure 4. Volume dependence of the anomaly integral for $a m=0.003$ and $10 \leq L \leq 130$ for $r= \pm 1$ and $r$ averaged for naive Wilson with PBC.

$$
\begin{aligned}
2 m\left\langle\bar{\psi}_{x} \gamma_{5} \psi_{x}\right\rangle= & 2 g^{2} \epsilon_{\mu \nu \rho \lambda} \operatorname{trace} F_{\mu \nu}(x) F_{\rho \lambda}(x) \frac{1}{(2 \pi)^{4}} \sum_{p} \cos \left(p_{\mu} a\right) \cos \left(p_{\nu} a\right) \cos \left(p_{\rho} a\right) \\
& \times m\left[\cos \left(p_{\lambda} a\right)\left[m+W_{0}(p)\right]-4 \frac{r}{a} \sin ^{2}\left(p_{\lambda} a\right)\right]\left(\mathcal{G}_{0}(p)\right)^{3} \\
& =\frac{g^{2}}{8(\pi)^{4}} \epsilon_{\mu \nu \rho \lambda} \operatorname{trace} F_{\mu \nu}(x) F_{\rho \lambda}(x) \tilde{I}_{m}(a m, r, L)
\end{aligned}
$$

where

$$
\begin{aligned}
\tilde{I}_{m}(a m, r, L)= & \sum_{p} \cos \left(p_{\mu} a\right) \cos \left(p_{\nu} a\right) \cos \left(p_{\rho} a\right) \\
& \times m\left[\cos \left(p_{\lambda} a\right)\left[m+W_{0}(p)\right]-4 \frac{r}{a} \sin ^{2}\left(p_{\lambda} a\right)\right]\left(\mathcal{G}_{0}(p)\right)^{3} .
\end{aligned}
$$

First by using Reisz power counting theorem [20] we show that the PSD term to $\mathcal{O}\left(g^{2}\right)$ is independent of $m$ in the infinite volume continuum limit. In the limit $L \rightarrow \infty$

$$
\tilde{I}_{m}(a m, r)=\int_{-\frac{\pi}{a}}^{\frac{\pi}{a}} d^{4} p \frac{N(m, p, a)}{D(m, p, a)}
$$


where

$$
\begin{aligned}
N(m, p, a) & =\cos \left(p_{\mu} a\right) \cos \left(p_{\nu} a\right) \cos \left(p_{\rho} a\right) \times m\left[\cos \left(p_{\lambda} a\right)\left[m+W_{0}(p)\right]-4 \frac{r}{a} \sin ^{2}\left(p_{\lambda} a\right)\right] \\
& =\cos \left(p_{\mu} a\right) \cos \left(p_{\nu} a\right) \cos \left(p_{\rho} a\right) \times m\left[\cos \left(p_{\lambda} a\right)\left[m+\frac{r}{a} \sum_{\mu}\left[1-\cos \left(a p_{\mu}\right)\right]\right]-4 \frac{r}{a} \sin ^{2}\left(p_{\lambda} a\right)\right] \\
D(m, p, a) & =\mathcal{G}_{0}(p)^{-3}=\left(\frac{1}{a^{2}} \sum_{\mu} \sin ^{2}\left(a p_{\mu}\right)+\left(m+\frac{r}{a} \sum_{\mu}\left[1-\cos \left(a p_{\mu}\right)\right]\right)^{2}\right)^{3}
\end{aligned}
$$

To find lattice degree of divergences (LDD) of $N(m, p, a)$ and $D(m, p, a)$ we scale $p$ and $a$ in the following way such that $\hat{p}=p a$ remains fixed,

$$
\begin{aligned}
& p \rightarrow \lambda p \\
& a \rightarrow a / \lambda .
\end{aligned}
$$

Then,

$$
\begin{aligned}
N(m, p \lambda, a / \lambda) & =\frac{r}{a}\left[m \sum_{\mu}\left[1-\cos \left(a p_{\mu}\right)\right]-4 \sin ^{2}\left(p_{\lambda} a\right)\right] \lambda+\mathcal{O}\left(\lambda^{0}\right) \\
D(m, p \lambda, a / \lambda) & =\left[\frac{1}{a^{6}}\left[\sum_{\mu} \sin ^{2}\left(a p_{\mu}\right)\right]^{3}+\frac{r^{6}}{a^{6}}\left[\sum_{\mu}\left[1-\cos \left(a p_{\mu}\right)\right]\right]^{6}+\frac{3 r^{2}}{a^{6}}\left[\sum_{\mu} \sin ^{2}\left(a p_{\mu}\right)\right]^{2}\left[\sum_{\mu}\left[1-\cos \left(a p_{\mu}\right)\right]\right]^{2}\right. \\
& \left.+\frac{3 r^{4}}{a^{6}}\left[\sum_{\mu} \sin ^{2}\left(a p_{\mu}\right)\right]\left[\sum_{\mu}\left[1-\cos \left(a p_{\mu}\right)\right]\right]^{4}\right] \lambda^{6}+\mathcal{O}\left(\lambda^{5}\right)
\end{aligned}
$$

Then according to Reisz, LDD of $N\left(\operatorname{degr}_{\hat{p}} N\right)$ and LDD of $D\left(\operatorname{degr}_{\hat{p}} D\right)$ are given by,

$$
\begin{aligned}
& \operatorname{degr}_{\hat{p}} N=1 \\
& \operatorname{degr}_{\hat{p}} D=6
\end{aligned}
$$

So, LDD of the integral in Eq. (6.4) is

$$
4+1-6=-1<0
$$

Then according to the Reisz power counting theorem, continuum limit of $\tilde{I}_{m}(a m, r)$ is

$$
\begin{gathered}
\int_{-\infty}^{\infty} d^{4} p \lim _{a \rightarrow 0}\left[\cos \left(p_{\mu} a\right) \cos \left(p_{\nu} a\right) \cos \left(p_{\rho} a\right) \times m\left[\cos \left(p_{\lambda} a\right)\left[m+W_{0}(p)\right]-4 \frac{r}{a} \sin ^{2}\left(p_{\lambda} a\right)\right]\left(\mathcal{G}_{0}(p)\right)^{3}\right] \\
=\int_{-\infty}^{\infty} d^{4} p \frac{m^{2}}{\left(p^{2}+m^{2}\right)^{3}}=\frac{\pi^{2}}{2} . \\
\tilde{I}_{m}(a m, r, L)=-\frac{2}{\pi^{2}} I_{m}(a m, r, L) \\
\lim _{a \rightarrow 0, L \rightarrow \infty} I_{m}(a m, r, L)=-1
\end{gathered}
$$

and hence $\lim _{a \rightarrow 0, L \rightarrow \infty} I_{m}(a m, r, L)=-1$ is independent of $m$. 
For the PSD term when am decreases one needs to go to larger value of $L$ compared to the anomaly term to achieve the infinite volume limit. It is important to note that as long as am is nonzero, there is no infrared singularity. This is borne out by numerical calculations as shown in Fig. 5(b). Furthermore we see that the lattice result differs from the continuum result by exhibiting considerable am dependence (see Fig. 5(a)) which appears to be a manifestation of cutoff effects with naive Wilson fermion. Nevertheless we see that the two terms, namely, the anomaly term and the PSD term to $\mathcal{O}\left(g^{2}\right)$ cancel each other independent of the value of am for large enough volumes. To check whether $r$ averaging can remove the cutoff effects we study the am dependence of the $r$ averaged PSD term. Fig. 5(c) shows that the PSD term is almost independent of am (for am $<=.1$ ) as in the continuum when $r$-averaging is performed.

\section{Conclusions}

In this work we have addressed four issues related to the one loop lattice calculation of chiral anomaly in the case of Wilson-like fermions.

Some general remarks are in order. The weak coupling perturbative analysis may not be sensitive to some lattice artifacts that may be present in numerical simulations. On the other hand, some of the cutoff effects found in the perturbative analysis especially for the naive Wilson fermions could be removed by smoothing of gauge fields and smearing of hadronic operators in numerical simulations.

We have used one loop lattice calculation of chiral anomaly to study quantitatively the effects of $r$ averaging which has been proposed before to achieve better scaling behavior of observables with Wilson fermions. We find that $r$ averaging of naive Wilson fermion has a slightly better approach to continuum chiral limit compared to OStm Wilson fermions. We show that in this case $r$ averaged result is much better than $\mathcal{O}(a)$ and even than $\mathcal{O}\left(a^{2}\right)$ improved Wilson fermion with tree level coefficients. It is the physical fermion contribution which is largely influenced by the $r$ averaging as seen in Fig. 2(d).

We have studied the doubler contributions as a function of $r$ and lattice fermion mass am. We have verified that the doubler contribution decreases as $r$ increases and it is not very sensitive to the lattice fermion mass.

Next, we have studied the possible interplay between finite size and cutoff effects by investigating in detail naive, $\mathcal{O}(a)$ improved and OStm Wilson fermion cases for a range of volumes and $a m$. We find that the major contribution to the interplay between finite volume and cutoff effects is at the $\mathcal{O}(a)$ level for naive Wilson fermion.

Lastly we have studied the relative roles played by the PSD term and the anomaly term in the flavor singlet axial Ward Takahashi identity for naive Wilson fermion on the lattice at one loop level for slowly varying background gauge fields. First by using Reisz power counting theorem we have shown that the PSD term to $\mathcal{O}\left(g^{2}\right)$ is independent of $m$ and cancels the anomaly term in infinite volume continuum limit. The lattice result for PSD term differs from the continuum result by exhibiting considerable am dependence for $r=+1$ or $r=-1$ (see Figs. 5(a) and 5(c)) which appears to be a manifestation of cutoff effects with naive Wilson fermion. Nevertheless we find that the two terms, namely, the 
(a)

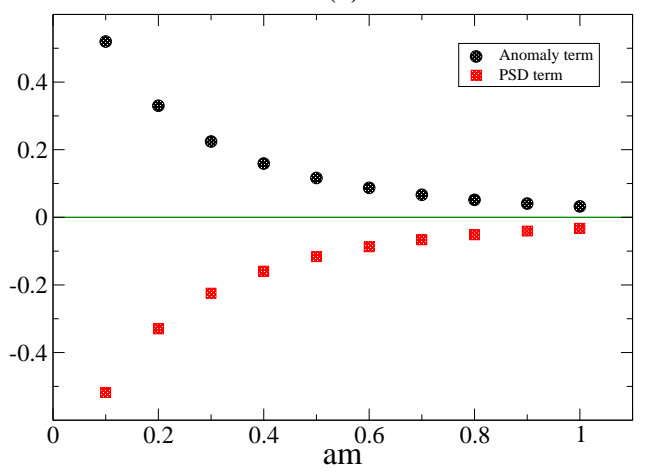

(b)

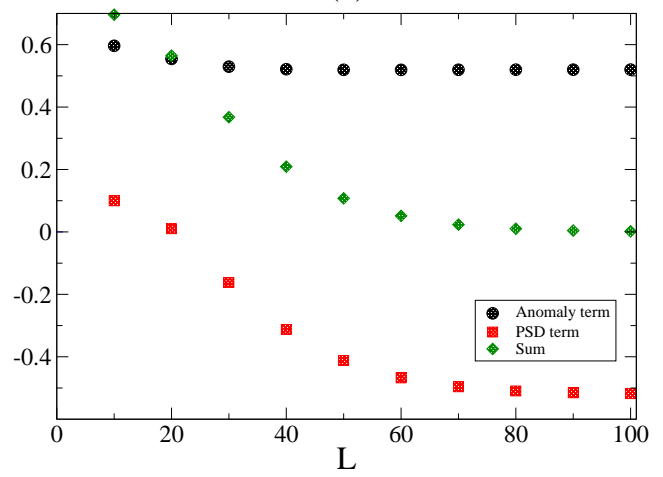

(c)

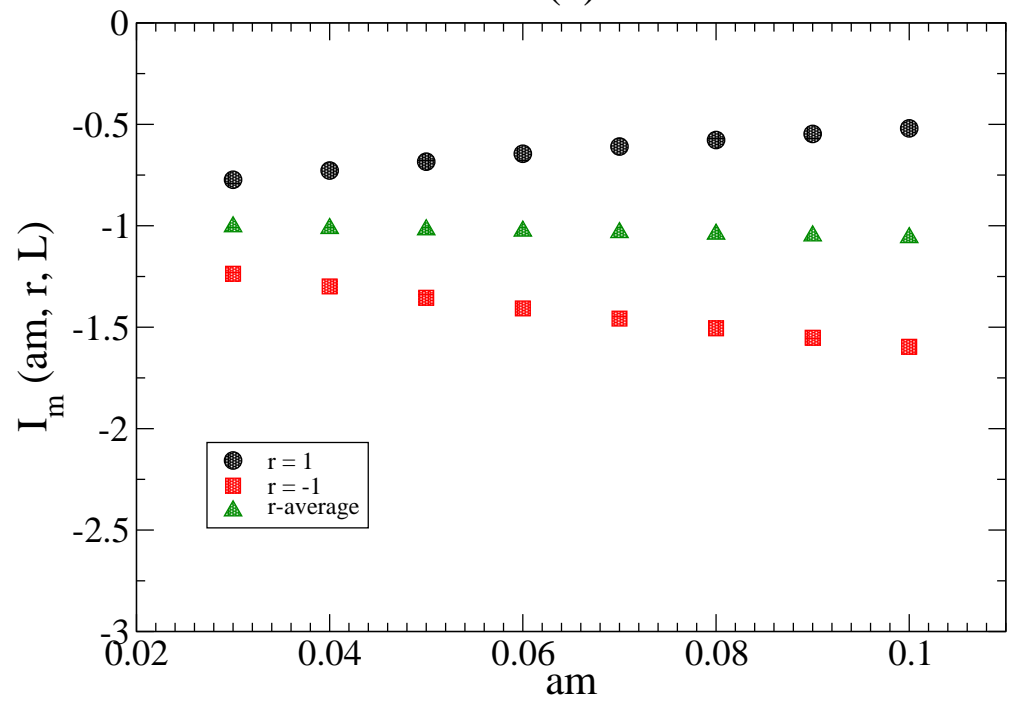

Figure 5. (a) The am dependence of the anomaly term and the PSD term for $L=100$ at $\mathcal{O}\left(g^{2}\right)$. (b) The $L$ dependence of the anomaly term, the PSD term and the sum for $a m=0.1 \mathcal{O}\left(g^{2}\right)$. (c) The PSD term for $r= \pm 1$ and the average, for $0.03 \leq a m \leq 0.2$ and $L=300 \mathcal{O}\left(g^{2}\right)$.

anomaly term and the PSD term to $\mathcal{O}\left(g^{2}\right)$ cancel each other independent of the value of am for $r=+1$ or $r=-1$ for large enough volumes. To verify whether $r$ averaging can remove the cutoff effects we investigate the am dependence of the $r$ averaged PSD term. Fig. 5(c) shows that the PSD term is almost independent of am when $r$-averaging is performed.

In the light of the results obtained above, it is tempting to try out $r$-averaging in lattice QCD simulations with naive Wilson fermions. However, problems related with unphysical UV fluctuations may potentially make the simulation difficult. Smearing of gauge links in 
the molecular dynamics trajectories has been used successfully to damp these unphysical fluctuations [21, 22].

\section{References}

[1] A. K. De, A. Harindranath and S. Mondal, Phys. Lett. B 682, 150 (2009) [arXiv:0910.5611 [hep-lat]].

[2] K. Osterwalder and E. Seiler, Annals Phys. 110, 440 (1978).

[3] K. G. Wilson, "Quarks and Strings on a Lattice", in New Phenomena in Subnuclear Physics, proceedings of the International School of Subnuclear Physics, Erice, 1975, edited by A. Zichichi (Plenum, New York, 1977).

[4] H. W. Hamber and C. M. Wu, Phys. Lett. B 133, 351 (1983); H. W. Hamber and C. M. Wu, Phys. Lett. B 136, 255 (1984).

[5] W. Wetzel, Phys. Lett. B 136, 407 (1984).

[6] T. Eguchi and N. Kawamoto, Nucl. Phys. B 237, 609 (1984).

[7] L. H. Karsten and J. Smit, Nucl. Phys. B 183, 103 (1981).

[8] W. Kerler, Phys. Rev. D 23, 2384 (1981).

[9] Also see, W. Kerler, Int. J. Mod. Phys. A 16, 3117 (2001) [arXiv:hep-lat/0007023].

[10] E. Seiler and I. O. Stamatescu, Phys. Rev. D 25, 2177 (1982) [Erratum-ibid. D 26, 534 (1982)].

[11] S. Aoki, Phys. Rev. D30, 2653 (1984);

[12] F. David and H. W. Hamber, Nucl. Phys. B248, 381 (1984);

[13] R. Frezzotti, G. C. Rossi, JHEP 0408, 007 (2004). [hep-lat/0306014].

[14] R. Frezzotti, Nucl. Phys. Proc. Suppl. 119, 140 (2003) [arXiv:hep-lat/0210007].

[15] S. Sint, arXiv:hep-lat/0702008.

[16] A. Shindler, Phys. Rept. 461, 37 (2008) [arXiv:0707.4093 [hep-lat]].

[17] R. Baron et al. [ ETM Collaboration ], JHEP 1008, 097 (2010) [arXiv:0911.5061 [hep-lat]] and references therein.

[18] P. Dimopoulos, R. Frezzotti, C. Michael, G. C. Rossi, C. Urbach, Phys. Rev. D81, 034509 (2010) [arXiv:0908.0451 [hep-lat]].

[19] H. J. Rothe and N. Sadooghi, Phys. Rev. D58, 074502 (1998). [hep-lat/9803026].

[20] T. Reisz, Commun. Math. Phys. 117, 79 (1988); T. Reisz, Commun. Math. Phys. 116, 573 (1988).

[21] S. Capitani, S. Durr, C. Hoelbling, JHEP 0611, 028 (2006) [hep-lat/0607006].

[22] S. Durr, Z. Fodor, C. Hoelbling, R. Hoffmann, S. D. Katz, S. Krieg, T. Kurth, L. Lellouch, T. Lippert, K. K. Szabo and G. Vulvert, Phys. Rev. D79, 014501 (2009) [arXiv:0802.2706 [hep-lat]]. 